\title{
Giant Lipoma of the Head and Neck Region: Case Report and Review of the Literature
}

\author{
Risimati Ephraim Rikhotso*, Gugulethu Mhlanga, Muhammad Bobat \\ Department of Maxillofacial and Oral Surgery, University of the Witwatersrand, Johannesburg, South Africa \\ Email: *erikhotso@gmail.com
}

How to cite this paper: Rikhotso, R.E. Mhlanga, G. and Bobat, M. (2017) Giant Lipoma of the Head and Neck Region: Case Report and Review of the Literature. Open Journal of Stomatology, 7, 469-474. https://doi.org/10.4236/ojst.2017.711042

Received: September 4, 2017

Accepted: November 7, 2017

Published: November 10, 2017

Copyright $\odot 2017$ by authors and Scientific Research Publishing Inc. This work is licensed under the Creative Commons Attribution International License (CC BY 4.0).

http://creativecommons.org/licenses/by/4.0/

\begin{abstract}
Head and neck lipomas are fairly common but their presentation in areas like submandibular, submental and sublingual regions are rarely seen. Lipomas are generally asymptomatic and gradually progressive in size, causing aesthetic and functional deficits. Surgical excision remains the mainstay of treatment. Radiological investigations like CECT and MRI play a critical role in diagnosis and management of these lesions. We describe a case of a giant lipoma involving the sublingual, submandibular and submental region, and its management.
\end{abstract}

\section{Keywords}

Lipoma, Head and Neck, Submandibular, Sublingual, Submental Spaces

\section{Introduction}

Lipomas are slow growing, nearly always benign adipose tumours that usually present subcutaneously. They are the most common neoplasms of mesenchymal origin [1]. They can occur anywhere in the body where fat is normally present. Lipomas present most commonly in the $50^{\text {th }}$ or $60^{\text {th }}$ decade of life and are multiple in $5 \%$ of patients but are uncommon in children [2]. Thirteen percent of all lipomas are located in the head and neck region [1]. They constitute $4.4 \%$ of all intra-oral tumours, with the cheek being the most common location [3]. Lipomas occurring in the submandibular and submental regions are extremely rare [4]. We report an unusually large combined intra- and extra-oral lipoma. Review of the literature and its treatment are also discussed.

\section{Case Report}

A 52-year-old African female presented to the Maxillofacial and Oral Surgery outpatient clinic with a chief compliant of slow growing, non-painful 4 year old 
swellings on the right side of her neck and also under the chin. She also complained of an enlarging asymptomatic mass in the mouth under her tongue. Patient reported that the swellings recently interfered with eating, speech and swallowing. No history of trauma, toothache, fever or weight loss was reported by the patient. The patient's medical history was significant for hypertension, which was well-controlled.

Physical examination revealed non-tender, fluctuant to doughy swelling on the right submandibular and submental areas. The swelling was extending from the right lower border of the mandibular to the level of the thyroid cartilage and from the angle to the body, about $3 \mathrm{~cm}$ beyond normal facial contour and overlying skin appeared normal (Figure 1). The second swelling located on the submental area extended from just behind the lower border of the mandible to the level of the hyoid bone, and was about $2 \mathrm{~cm}$ over normal contour. No discharge or palpable lymphadenopathy was noted.

Intra-oral examination (Figure 2) revealed a non-tender doughy swelling in the right floor of the mouth with resultant displacement of the tongue superiorly and to the contralateral side. Flow of saliva was normal. Overlying mucosa general appeared normal except in the area which presented with an ulcer associated with fractured non-carious 46 (which suggested a traumatic ulcer) and an area bellow the tip of the tongue with a yellowish tinge. Bimanual palpation confirmed continuity with the extra-oral submandibular and submental swellings.

Panoramic radiograph was taken and excluded odontogenic source of the le-

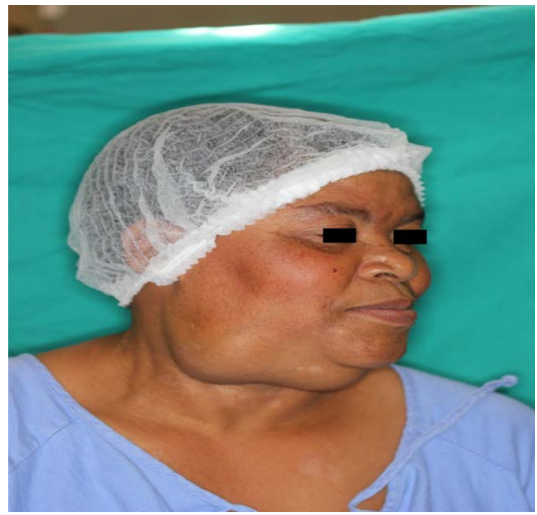

Figure 1. Photograph showing a bilobulated, submandibular and submental swelling.

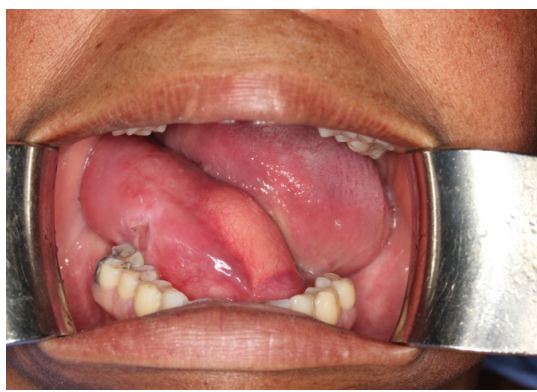

Figure 2. Intra-oral image showing floor of the mouth swelling. 
sion. A clinical differential diagnosis of a plunging ranula was made. Fine needle aspiration cytology from the swelling was inconclusive. Contrast-enhanced computed tomography (CECT) showed (Figure 3) a non-enhancing, hypodense, homogenous lesion with minimal septation located on the sublingual area extending anteriorly from the genial area and posteriorly to the submandibular space. The lesion also extended to the submental space (Figure 4), and was suggestive of a lipoma or a liposarcoma. Plunging ranula was then replaced by lipoma/liposarcoma as the most likely diagnosis of the lesion.

\section{Treatment}

Intra-operatively, an orange needle was used to aspirate the sublingual swelling, and no fluid or saliva was aspirated. The lesion was dissected bluntly and removed in its entirety from the sublingual, submandibular and submental regions via an intra-oral incision made in the floor of the mouth (Figure 5). The excised smooth, lobulated, greasy to touch, yellowish to orange coloured lesion (Figure 6) was submerged in formalin and floated, confirming the diagnosis of lipoma. Lesion was also confirmed as a lipoma histopathologically, consisting of mature fat cells without lipoblasts or any feature of malignancy (Figure 7).



Figure 3. Axial CT scan showing a non-enhancing, homogenous lesion with septation extending anteriorly from the genial area to the submandibular area posteriorly.

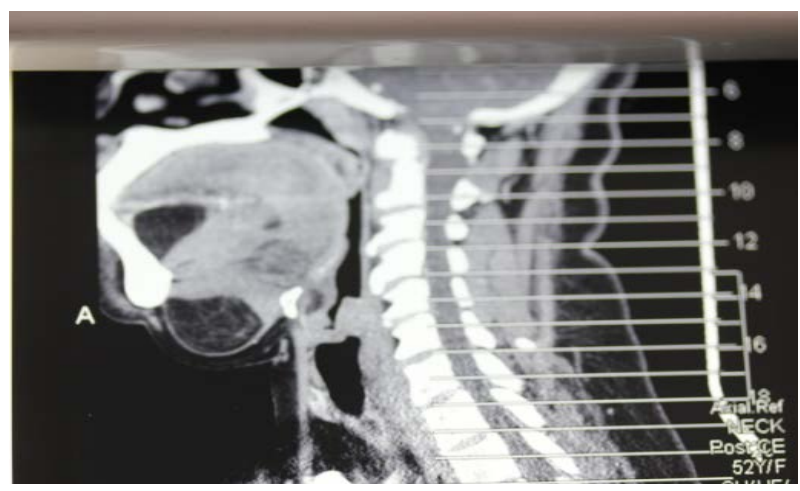

Figure 4. Sagittal CT Scan showing the lesion in the sublingual and submental spaces. Septation is clearly visible in the submental space. 




Figure 5. Intra-operative image showing excision of tumour.

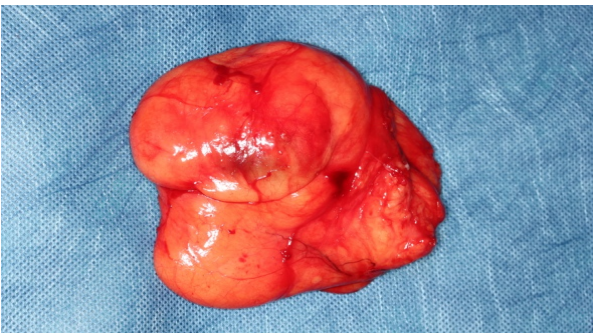

Figure 6. Excised tumour specimen sent for histopathology.

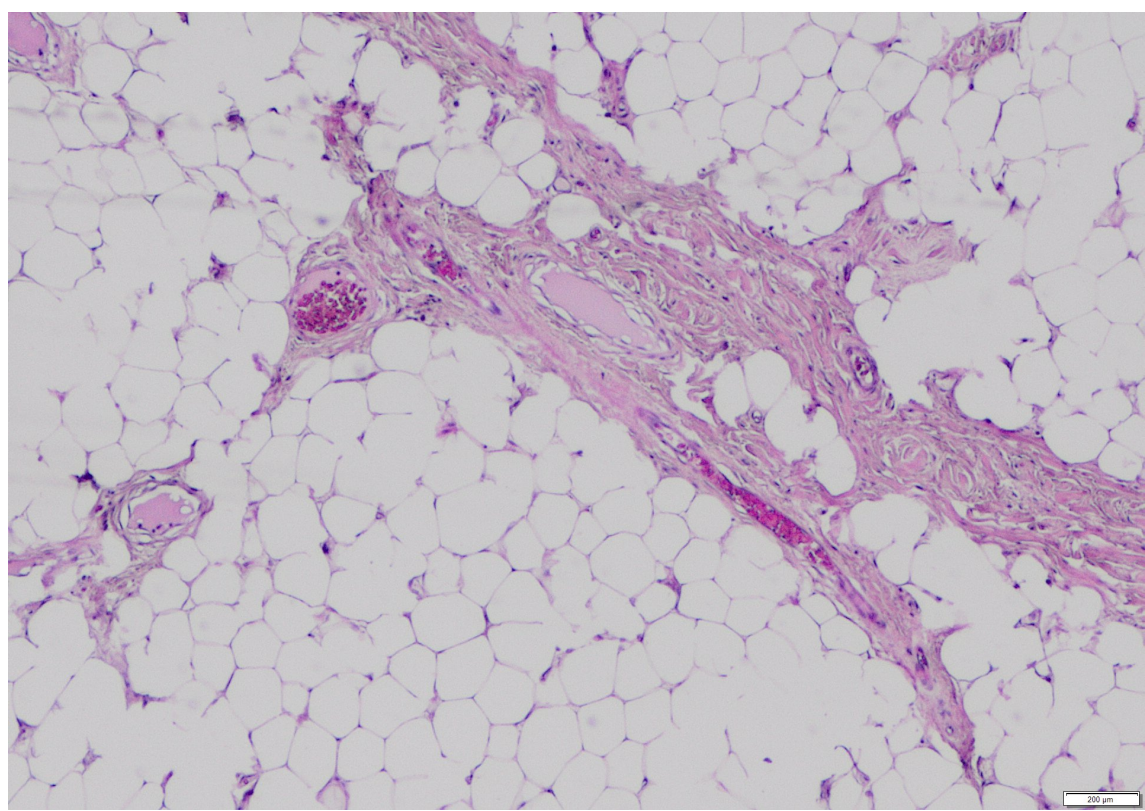

Figure 7. Histology showing mature fat cells without lipoblasts.

Follow-up visits were performed at 1 week, 3, 6, 12 and 24 months. The patient had healed uneventfully and there was no evidence of recurrence at the 2 year follow-up visit.

\section{Discussion}

Head and neck lipomas are fairly common but their presentations in sites such as the submandibular, sublingual and submental regions are rarely seen. The 
rarity of their presentation in these sites can often lead to difficulties in making clinical diagnosis.

Lipomas are generally asymptomatic and gradually progressive in size, causing aesthetic and functional deficits such as difficulty in chewing and speech (as exemplified in our patient).

One way of classifying lipomas is on the basis of their weight or size. A tumour is classified as a giant lipoma if the size is greater than $100 \mathrm{~mm}$ in one dimension or the weight is greater than $1000 \mathrm{~g}$ [4] [5]. The specimen in our patient weighed $125 \mathrm{~g}$ and measured $105 \times 50 \times 40 \mathrm{~mm}$.

Obesity and local growth of adipose tissues (hypertrophy theory) may both be responsible for formation of a lipoma [6]. The metaplastic theory holds the view that lipomatous development represents abnormal differentiation in situ of mesenchymal cells into lipoblasts [7]. Trauma is also thought to play a role in development of lipomas [8]. Our patient had no history of trauma but was morbidly obese. The clinical presentation profile of our patient was highly suggestive of a plunging ranula. Perhaps this case illustrates that any clinical diagnosis of a plunging ranula should always be accompanied by supplemental diagnostic tools such as FNA, ultrasound, CT scans and sometimes MRIs to confirm the cystic nature of the lesion and to exclude lesions such as lipomas. Ultrasound was not done in our patient. Lipomas are reported to appear as hyperechoic elliptical masses parallel to the skin surface and contains linear echogenic lines at right angles to the ultrasound beam [1].

Both CTs and MRIs are imaging modalities that can be used to confirm preoperative diagnosis of lipomas. CT, as was the case with our patient, will show a well encapsulated homogeneous mass with few septations and typically low CT attenuation number with values of -50 to -150 Hounsfield units [3] [8]. (Fat is the only soft tissue with a density less than water, and has a low CT attenuation number). MRI is the modality of choice for imaging lipomas, not only to confirm the diagnosis, which is usually strongly suggested by ultrasound and CT but also to accurately determine exact size, location and extension. MRIs also help delineate the surrounding soft tissues from the lesion (lipoma) [8] [9]. Lipomas follow subcutaneous fat signal on all sequences, and demonstrate high signal intensity on T1 weighted images and T2 weighted fast spin echo MRI sequences [8]. Waiting list longer than six months precluded acquisition of an MRI for our patient. It is important to understand that neither CT nor MRI can differentiate a lipoma from a liposarcoma. Only histological examination can make this distinction with certainty. Liposarcomas rarely arise from pre-existing lipomas and mostly arise de novo.

Surgical excision is the mainstay treatment of lipomas and recurrences after adequate excision are very rare. A high index of suspicion for a liposarcoma should be raised with any recurrence of a lipoma. For our patient postoperative recovery was uneventful, and no recurrence was seen during a 24 months follow-up. 


\section{Conclusion}

We report on a rare case of a giant solitary lipoma with intra- and extra-oral extension with clinical features of a plunging ranula. To the best of our knowledge this is the first reported case of simultaneous involvement of sublingual, submental and submandibular regions by a lipoma

\section{Conflict of Interest}

None.

\section{Compliance with Ethical Standards}

Informed consent was obtained from the patient.

\section{References}

[1] Ahuja A.T., King, A.D., Ken, J., King, W. and Metrerveli, C. (1998) Head and Neck Lipomas: Sonographic Appearance. American Journal of Neuroradiology, 19 , 505-508.

[2] Kransdorf, M.J. (1995) Bening Soft-Tissue Tumours in a Large Referral Population: Distribution of Specific Diagnosis by Age, Sex and Location. AJR American Journal Roentgenology, 164, 395-402. https://doi.org/10.2214/ajr.164.2.7839977

[3] Daltilo, D., Taiige, J. and Nwanas, E.J. (1996) Intraoral Lipoma of the Tongue and Submandibular Space: Report of a Case. Journal of Oral and Maxillofacial Surgery, 54, 915.

[4] Sanchez, M.R., Golomb, F.M., Moy, J.A. and Ptozkin, J.R. (1993) Giant Lipoma: Case Report and Review of the Literature. Journal of the American Academy of Dermatology, 28, 266-268. https://doi.org/10.1016/S0190-9622(08)81151-6

[5] Kalia, V., kaushal, N. and Pahwa, D. (2011) Giant Subcutaneous Solitary Lipoma Arising in the Neck: Case Report and Review of Literature. Webmed Central Maxillofacial Surgery, 2, WMC001838.

[6] Das Gupta, T.K. (1978) Tumours and Tumour-Like Conditions of Adipose Tissues. Current Problems in Surgery, 1, 1.

[7] El-Monem, M.H., Gaafar, A.H. and Magdy, E.A. (2006) Lipomas of the Head and Neck: Presentation Variability and Diagnostic Workup. The Journal of Laryngology \& Otology, 120, 47-55.

[8] Signorini, M. and Cmpiglio, G.L. (1998) Posttraumatic Lipoma: Where Do They Really Come from? Plastic and Reconstructive Surgery, 101, 699-705.

[9] Gaskin, C.M. and Helms, C.A. (2004) Lipomas, Lipoma Variants, and WellDifferentiated Liposarcomas (Atypical Lipomas): Results of MRI Evaluations of 126 Consecutive Fatty Masses. AJR. American Journal of Roentgenology, 182, 733-739. https://doi.org/10.2214/ajr.182.3.1820733 